Mediterránea Ser. Biol. (1988), n. ${ }^{\circ}$ 10. Pág. 63-85

\title{
BALANCES DE NUTRIENTES EN PEQUEÑAS CUENCAS DE ENCINAR. II. QUIMISMO DE LA PRECIPITACIÓN Y APORTES DE ORIGEN ATMOSFÉRICO
}

\author{
por \\ JUAN BELLOT ${ }^{1}$ y ANTONIO ESCARRÉ
}

\section{RESUMEN}

Durante un período de tres años, se ha estudiado el quimismo del agua de lluvia y se han estimado los aportes de nutrientes en la deposición global a una cuenca de encinar en la serra de Prades (Tarragona, España). En ninguno de los iones analizados se ha observado diferencia significativa entre las muestras colectadas en dos altitudes distintas: 700 y $950 \mathrm{~m}$. s. n. m.

La precipitación es moderadamente ácida $(\mathrm{pH}=4.96)$ y los iones más importantes en la lluvia son $\mathrm{SO}_{4}^{2-}$ y $\mathrm{Ca}^{2+}$, que representan el 23.6 y el $18.4 \%$ del total, respectivamente. La influencia marina se hace patente por las elevadas proporciones de $\mathrm{Cl}$ y Na. En Prades, más del $95 \%$ de estos iones son, presumiblemente, de origen marino.

El seguimiento de series de lluvias producidas en un corto lapso de tiempo ha permitido poner de manifiesto la existencia de elementos neutralizadores en el polvo y aerosoles atmosféricos, que se agotan en las primeras precipitaciones. Consiguientemente, en un $84 \%$ de las series estudiadas ha sido posible observar un descenso del $\mathrm{pH}$ en las muestras de lluvias sucesivas.

El aporte total de $\mathrm{SO}_{4}^{2-}(20 \mathrm{~kg} / \mathrm{ha})$ es más del doble de los aportes de otros iones $\left(\mathrm{Cl}^{-}\right.$, $\mathrm{NO}_{3}^{-}, \mathrm{Ca}^{2+}, \ldots$. La entrada de nitrógeno se ha estimado en $5 \mathrm{~kg} / \mathrm{ha} / \mathrm{año}$, lo que viene a representar algo menos del $20 \%$ del nitrógeno que se acumula anualmente en la vegetación.

\section{SUMARY}

The chemical characteristics of rain water and the amount nutrients received from the atmosphere were estudied for three years in an evergrenn-oak forest (Quercus ilex ssp.ilex) in the Prades mountains (Tarragona, Spain). Statistical treatment of data makes possible to confirm the lack of significative differences between the rainfall chemistry in two altitudes: 700 and $950 \mathrm{~m}$. o.s.l.

The precipitation is moderately acid $(\mathrm{pH}=4.96)$ and the most importants ions in rainfall are $\mathrm{SO}_{4-}^{2-}$ and $\mathrm{Ca}^{2+}$, which represent 23.6 and $18.4 \%$ of total. Both ions reflect the importance of

(1) Instituto Agronómico Mediterráneo, Zaragoza.

(2) Departamento de Ciencias Ambientales y Recursos Naturales, Universidad de Alicante. 
continental dust in the dry deposition at Prades. The marine influence is indicated by high proportions of $\mathrm{Cl}^{-}$and $\mathrm{Na}^{2+}$. In Prades more than $95 \%$ of them have this source.

The monitoring of series of continuos rainfalls seems to reflect that the neutralization capacity of the «washout» is limited in this region. In $84 \%$ of the estudied series two or three events are sufficient to reduce the initial $\mathrm{pH}$ level to the acidity theoretically characteristic of the «rainout».

The annual input of $\mathrm{SO}_{4}^{2-}(20 \mathrm{~kg} / \mathrm{ha})$ is more than twice as much as the input of other $(\mathrm{Cl}-$, $\mathrm{NO}_{3}^{-}, \mathrm{Ca}^{2+}, \ldots$ ). The nitrogen input wasevaluated in $5 \mathrm{~kg} / \mathrm{ha} / \mathrm{yr}$ and probably represents less than $20 \%$ of annual acumulation in plants.

\section{INTRODUCCIÓN}

En un trabajo previo con el mismo título preliminar (ESCARRÉ et al. 1982) se trataba el proceso seguido para la selección del complejo de cuencas de encinar del Monte Poblet (Serra de Prades) como las más aptas para su estudio biogeoquímico, y también el sistema utilizado para la instrumentalización de una de ellas, el barranc de l'Avic.

Desde la publicación de aquel estudio se han producido interesantes aportaciones al conocimiento de los ciclos de nutrientes en el encinar mediterráneo, tanto en la dehesa salmantina (ESCUDERO et al. 1985), como en un bosque montano del Montseny (La Castanya), en el que el desfronde y la descomposición han sido tratados por VERDÚ (1984) y la producción primaria y el almacenamiento de nutrientes en la vegetación por FERRÉS (1984). Los aspectos referentes a los aportes externos con la precipitación han merecido especial atención por parte de RODÁ (1983) y ÁVILA (1986). Un intento de síntesis de todos estos procesos en el encinar y su comparación con otros ecosistemas forestales se presenta en FERRÉS et al. (1984) y ESCARRÉ et al. (1986).

También sobre el encinar del Monte Poblet, que presenta rasgos de mediterraneidad más acusados que el de la Castanya, han aparecido algunos trabajos preliminares que lo estudian en una unidad cuenca, el barranc de l'Avic [ESCARRÉ et al. 1983; ESCARRÉ et al. 1984; LLEDÓ y ESCARRÉ (1984), ESCARRÉ et al. (1986) y PIÑOL et al. (en prensa)].

A pesar de los precedentes citados en el estudio de la química de la precipitación, la cantidad de información existente en este tema referida al área mediterránea es, aún hoy, muy reducida, por lo que resulta difícil valorar por este medio la importancia que la contaminación atmosférica de fondo tiene en esta área. El presente estudio pretende contribuir en este sentido a un mejor conocimiento de la química atmosférica y de su interacción con los ecosistemas forestales. Con él se contribuye en primer lugar a la descripción de las características más relevantes del quimismo de la precipitación en la estación experimental de l'Avic (grado de acidez, influencia marina, importancia de la deposición seca). En segundo lugar, se destacan las principales interrelaciones entre los iones presentes en el agua de lluvia, y por último se cuantifica la importancia de los aportes atmosféricos de los diferentes elementos en la precipitación. 


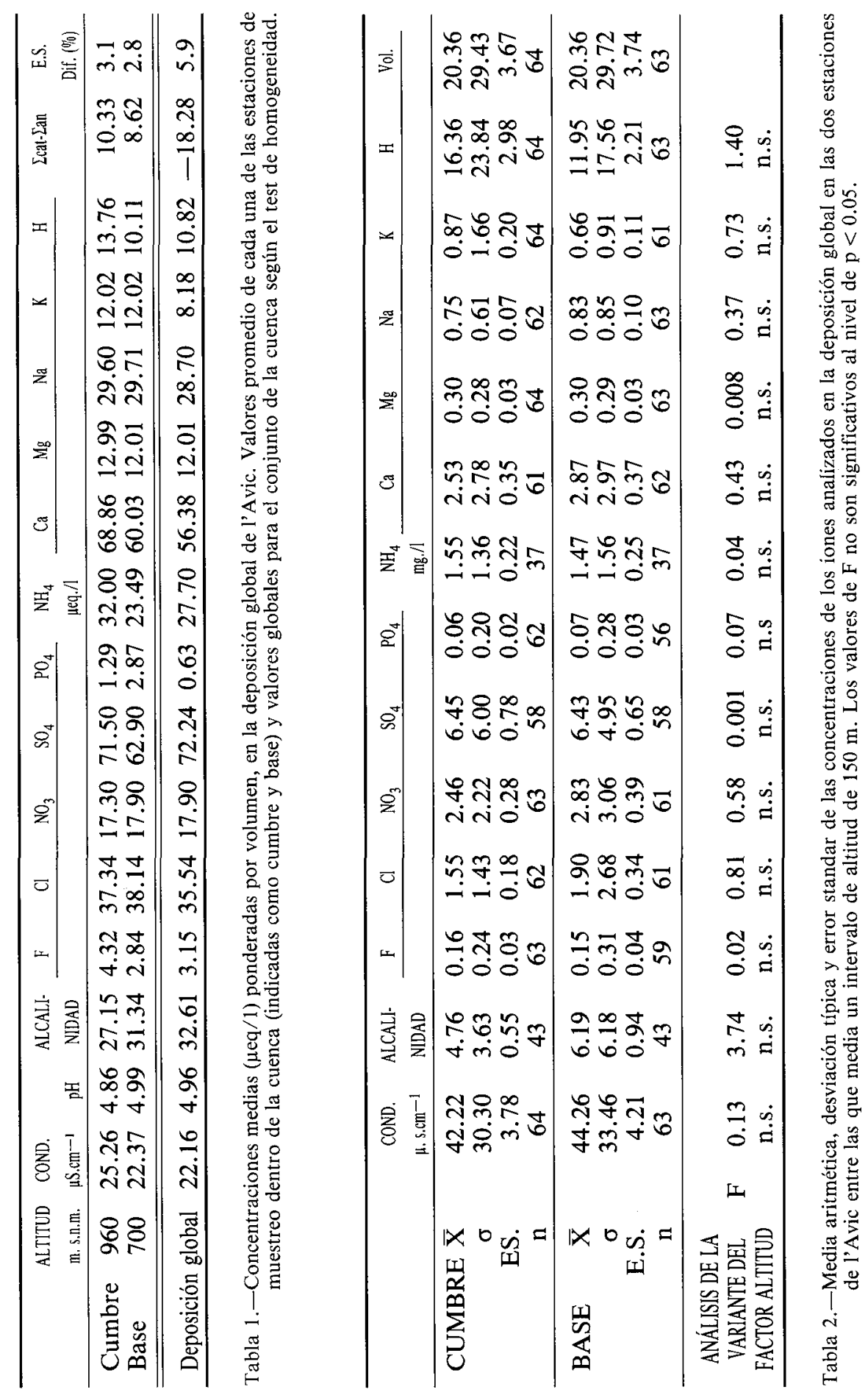




\section{METODOLOGÍA}

\section{ÁREA DE ESTUdIO}

El área de estudio está situada en el Monte Poblet (Sierra de Prades, Tarragona), en el complejo de cuencas descrito por ESCARRÉ et al. (1982). Los datos aquí aportados se tomaron en una cuenca de 55.5 ha. (Barranc de L'Avic), cuyas cotas altitudinales oscilan entre $\operatorname{los} 650$ y 1.100 m. s. n. m. La vegetación está constituida por un encinar sometido a carboneo 40 años atrás, lo que le confiere una estructura típica de rebrotes de zoca. Las especies arbóreas dominantes son la encina (Quercus ilex), el madroño (Arbutus unedo) y la falsa aladierna (Phillyrea media). Mayor información sobre las características del bosque y de la cuenca pueden encontrarse en ESCARRÉ et al. (1986).

\section{MATERIAL Y MÉTODOS}

Las entradas por precipitación se han controlado en dos estaciones situadas a 700 y 950 m.s.n.m. En cada una de ellas se disponía de 5 pluviómetros y 4 colectores de agua para análisis químicos, además de un pluviógrafo de registro continuo en la parte más baja. Los colectores para fines analíticos estaban formados por embudos de $30 \mathrm{~cm}$. de diámetro, situados a $1 \mathrm{~m}$. del suelo, y conectados a los depósitos a través de un bucle para evitar la evaporación (BORMANN et al. 1977). En todos los colectores se utilizó una malla plástica para evitar la deposición de insectos u otros materiales, y timol como biocida (SZABÓ, 1977). Los muestreos se realizaban tras cada precipitación, lavando los colectores «in situ» con abundante agua desionizada.

Como métodos analíticos, se han utilizado los electroquímicos para conductividad y $\mathrm{pH}$, y colorimétricos para alcalinidad y amonio (valoración con $\mathrm{H}_{2} \mathrm{SO}_{4}, 0.0002 \mathrm{~N}$, y reactivo de Nessler, respectivamente). Después, la muestra era filtrada con membrana de $45 \mu \mathrm{m}$ y se analizaban los aniones $\mathrm{F}^{-}, \mathrm{Cl}^{-}$, $\mathrm{NO}_{3}^{-}, \mathrm{SO}_{4}^{2-}$ y $\mathrm{PO}^{3-}$ por cromatografía iónica (Dionex 10), en tanto que los cationes $\mathrm{Ca}^{2+}, \mathrm{Mg}^{2+}, \mathrm{Na}^{+}$y K${ }^{+}$se medían por espectrofotometría de absorción atómica (Perkin Elmer Corporation, 1973).

Las concentraciones medias diarias se calculan mediante un análisis de homogeneidad entre las 8 muestras del día, tal como se describe en ESCARRÉ et al. (1986). Los valores mensuales y anuales de concentración se calculan ponderando las concentraciones por el volumen del período corespondiente.

\section{RESULTADOS Y DISCUSIÓN}

\section{Características de la precipitación}

El volumen de precipitación anual en la cuenca de L'Avic se ha medido entre los años 1981 y 1986, y se obtiene un valor medio de $553 \mathrm{~mm}$. El período es evidentemente corto para un clima mediterráneo, en el que la variación in- 
teranual es importante. Durante los dos años hidrológicos que cubrió este estudio las medidas de precipitación anual en la cuenca fueron 841 y $415 \mathrm{~mm}$.

La estación del Servicio Meteorológico Nacional más próxima al barranco de L'Avic es la de Riudabella, que está situada a 570 m s.n.m. y dista 3 $\mathrm{km}$ de la cuenca. Para esta estación el valor medio de los registros de precipitación en 20 años es de $590 \mathrm{~mm}$. En otras dos estaciones próximas, Montblanc y La Pena, situadas respectivamente a 350 y $875 \mathrm{~m}$ s.n.m., y a 12 y $5 \mathrm{~km}$ de L'Avic, las precipitaciones anuales medias durante 8 años son 560 y $800 \mathrm{~mm}$, respectivamente (FOLCH y VELASCO, 1974).

Aunque de estos datos se podría inferir la existencia de un claro gradiente altitudinal, la observación simultánea de dos estaciones en la misma cuenca de L'Avic, situadas a 700 y $950 \mathrm{~m}$ s.n.m., no han dado diferencias significativas mediante un test no paramétrico de Wilcoxon.

Para la descripción de los aspectos químicos de la precipitación, dada la importante relación existente en la concentración de los iones solubles en el agua de lluvia y el volumen de precipitación (por ej. GORHAM et al., 1958; LEMÉE, 1974), resulta una práctica usual reflejar la concentración promedio de cada ión, como la media ponderada por el volumen correspondiente al período de tiempo elegido. En la tabla 1, se presenta esta concentración iónica media, para todos los iones analizados, además de la conductividad en el agua de deposición global en l'Avic. Los iones dominantes son el $\mathrm{SO}_{4}^{2-}$ y el $\mathrm{Ca}^{2+}$, que constituyen el $23.6 \%$ y $18.4 \%$. de los equivalentes totales. El resto, suponen porcentajes inferiores, y entre ellos destacan $\mathrm{Cl}^{-}$, alcalinidad, $\mathrm{Na}^{+}$y $\mathrm{NH}_{4}^{+}$. En general, las precipitaciones promedio en L'Avic, son de aguas poco cargadas en sales solubles (cond $=22.1 \mu \mathrm{S} / \mathrm{cm}$ ) y moderadamente ácidas. $\mathrm{El} \mathrm{pH}$ medio es de 4.96, aunque se han registrado valores extremos de 3.9 y 7.45.

\section{Diferencias altitudinales}

Es importante el grado de representatividad que los valores de deposición global, obtenidos en estaciones concretas, tengan para todo el conjunto de la cuenca estudiada si el objetivo final es establecer balances de nutrientes a su nivel o definir los flujos de los mismos en el ecosistema.

En la tabla 1, se muestran los valores medios ponderados por volumen de las precipitaciones registradas en las dos estaciones establecidas. No se observan más que ligeras diferencias en las concentraciones, sobre todo los valores superiores de $\mathrm{SO}_{4}^{2-}, \mathrm{NH}_{4}^{+}$y F${ }^{-}$en la estación a mayor altura, y la alcalinidad y $\mathrm{Cl}^{-}$, algo superiores en la base de la cuenca.

REYNOLDS (1984), y ALTWICKER et al. (1986), abordan el problema de la representatividad mediante la comparación de las concentraciones medias calculadas en diferentes estaciones de muestreo en una o más cuencas, y en base a los errores standars de dichas medias. En la Tabla 2, se muestran los resultados obtenidos y se comprueba que las diferencias entre las concentraciones medias de ambas estaciones carecen de significación. Un análisis de la varianża, que utiliza la altitud como factor principal proporciona el mismo resultado (tabla 2). Estos resultados son relevantes ya que permiten utilizar concentraciones medias, estimadas con los datos de ambas estaciones, para caracterizar la química de la precipitación en la cuenca de l'Avic. En el caso con- 
trario, las estimas de trascolación y escorrentía cortical netas, se deberían calcular en base a concentraciones de precipitación tomadas a la altitud correspondiente de los árboles estudiados.

\section{Importancia de la deposición seca gravitacional en l'Avic}

EATON et al. (1980), SWANK (1984) y LINDBERG et al. (1986) entre otros, ponen de manifiesto que la contribución atmosférica a los ecosistemas terrestres, no sólo se efectúa por vía húmeda, sino que la deposición seca tiene una gran importancia cuantitativa como vía de entrada al ecosistema, que varía en función de los iones, las características topográficas, y la localización geográfica de la zona con respecto a fuentes emisoras. Sin embargo y a pesar de que para nutrientes tan importantes como $\mathrm{P}_{-} \mathrm{PO}_{4}^{3-}, \mathrm{N}_{-} \mathrm{NO}_{3}^{-}$y K${ }^{+}$, la vía seca puede llegar a representar entre el 64 y $90 \%$ del total anual (SWANK y HENDERSON, 1976), sólo en los últimos años ha proliferado los estudios que consideran por separado ambas entradas.

Aunque en este estudio no se ha podido muestrear la deposición húmeda con colectores especiales (GALLOWAY y LIKENS, 1976, 1978) se ha tratado de evaluar la importancia de la vía seca mediante una aproximación indirecta que parte de considerar que los recolectores abiertos no recogen todos los tipos de partíclas atmosféricas posibles (MESZAROS, 1981). Sólo la deposición gravitacional tendría lugar sobre estos colectores y no la impactación horizontal de aerosoles, cuyo diámetro oscila entre 0.1 y $10 \mu$. Estos últimos, que se desplazan por difusión y coagulan frente a obstáculos (MESZAROS, 1981), son captados con eficacia con los colectores de pantalla utilizados por MILLER y MILLER (1980). Con la finalidad de controlar este tipo de entradas, se instaló una antena con 4 de estos colectores. Durante el tiempo que permaneció instalada, se han podido calcular las tasas de incremento en las concentraciones iónicas con respecto a la deposición global. Estos valores indican que en l'Avic, las partículas que se impactan en esa pantalla y posteriormente son arrastradas y disueltas por el agua de lluvia, incrementan hasta 4.4 veces el valor del $\mathrm{Cl}^{-}, 3$ veces el del $\mathrm{NO}_{3}^{-}$, y entre 1.9 y 2.6 el del resto de iones, $\left(\mathrm{K}^{+}, \mathrm{F}^{-}\right.$, $\mathrm{Mg}^{2+}, \mathrm{SO}_{4}^{2-}, \mathrm{Na}^{+}$y $\mathrm{Ca}^{2+}$ ). SCHLESINGER y REINERS (1974), encuentran tasas entre 4.9 y 8.3 con procedimientos muy similares, y atribuyen un gran protagonismo a la existencia de vientos, nieblas o nubes a baja altura.

La importancia de la deposición seca, tiene especial interés si se intentan estimar los flujos de trascolación y escorrentía cortical netos (SCHELSINGER y HASEY, 1980), ya que se requiere para distinguir si los iones provienen del interior o exterior del sistema.

\section{Contribución marina a la precipitación}

Desde los trabajos pioneros de GAMBELL y FISHER (1966) y FISHER (1968), hasta el presente, son muchos los estudios que intentan determinar en qué medida el agua marina es responsable de la química de la precipitación. Las concentraciones de $\mathrm{Na}^{+} \mathrm{y} \mathrm{Cl}^{-}$en la precipitación pueden usarse a modo de índices de la importancia relativa del mar, como fuente de iones disueltos en la lluvia, y de aerosoles para los núcleos de las gótulas (GRANAT, 1978). 


\begin{tabular}{lllllllll}
\hline & $\mathrm{Cl} / \mathrm{Na}$ & $\mathrm{Mg} / \mathrm{Na}$ & $\mathrm{K} / \mathrm{Na}$ & $\mathrm{Ca} / \mathrm{Na}$ & $\mathrm{SO}_{4} / \mathrm{Na}$ & $\mathrm{Ca} / \mathrm{Mg}$ & REFERENCIA \\
\hline L'AVIC $\ldots \ldots .$. & 1.23 & 0.41 & 0.28 & 1.96 & 2.51 & 4.69 & Presente estudio \\
MONTSENY & 1.20 & 0.49 & 0.13 & 2.62 & 2.25 & 5.35 & Ávila (com. verb.) \\
MAR & $\ldots \ldots \ldots . .$. & 1.13 & 0.23 & 0.021 & 0.044 & 0.08 & 0.19 & Krauskop (1979) \\
\hline
\end{tabular}

Tabla 3.-Cocientes iónicos en el agua de la precipitación en las sierras de Prades y Montseny, junto a su valor en el agua del mar.

La concentración de $\mathrm{Na}^{+}$es con frecuencia ligeramente inferior a la que correspondería al $\mathrm{Cl}^{-}$presente, si se mantuviera la proporcionalidad marina. LIKENS et al. (1977) justifican esta diferencia porque parte del $\mathrm{Cl}^{-}$se deriva también de fuentes continentales generalmente antropogénicas. Si se admite que no existe un fraccionamiento de los iones de los aerosoles marinos durante su formación y penetración en el continente, y que todo el $\mathrm{Na}^{+}$presente en la lluvia proviene únicamente de dichos aerosoles, es posible estimar la contribución marina en otros iones, ya que con las suposiciones anteriores se mantendrían las proporciones iónicas del agua marina (GRANAT, 1978; SCHLESINGER et al., 1982; RODÁ, 1983).

Para l'Avic los cocientes entre la mayoría de los iones analizados y el $\mathrm{Na}^{+}$, figuran en la tabla 3. Se observa en todos ellos un enriquecimiento de origen continental en el agua de lluvia. Los cocientes $\mathrm{Ca}^{2+} / \mathrm{Na}^{+}, \mathrm{y} \mathrm{SO}_{4}^{2-} /$ $\mathrm{Na}^{+}$, indican claramente la existencia de una fuente continental de ambos.

En la fig. 1 se han representado los valores de la concentración de $\mathrm{Ca}^{2+}$, $\mathrm{Mg}^{2+}, \mathrm{K}^{+}, \mathrm{SO}_{4}^{2-}$ y $\mathrm{Cl}^{-}$, frente al $\mathrm{Na}^{+}$. Se puede ver que en todos los casos, excelpt en el $\mathrm{Cl}^{-}$, hay un aumento de estos iones frente a su nivel en el agua marina. $\mathrm{El}$ exceso de $\mathrm{SO}_{4}^{2-}$, es atribuido a descomposiciones orgánicas, las actividades antropogénicas (SCHLESINGER et al., 1982; LIKENS, 1981), y a las emisiones de dimetil sulfuro, producidas por el fitoplacton oceánico (BATES et al., 1987; CHARLSON et al., 1987). Los valores de $\mathrm{Mg}^{2+}$ y K${ }^{+}$, frente al $\mathrm{Na}^{+}$y sus cocientes, indican también enriquecimientos continentales en estos iones, aunque más moderados. Mientras que gran parte del $\mathrm{Mg}^{2+}$ es atribuido a los aerosoles marinos, el $\mathrm{K}^{+}$cuyo origen predominante es continental, puede deberse a fuentes antrópicas de carácter local (fertilizantes agrícolas por ejemplo), a partículas de suelo transportadas por el viento, a las cenizas (fly-ash) de combustión de carbones, o a los aerosoles biológicos (GOSZ, 1980).

Los valores de los cocientes encontrados en L'Avic son semejantes a los que RODÁ (1983) y ÁVILA (1986) citan para el encinar montano del Montseny, y que aparecen en la tabla 3.

Como se ha indicado antes, si se supone que todo el $\mathrm{Na}$ tiene procedencia marítima, se puede estimar qué proporción de los iones presentes en el agua de lluvia proceden del mar. La Tabla 4 presenta esta proporción para l'Avic y el Montseny. Las diferencias apenas merecen mencionarse y en general se aprecia que en ambas estaciones casi todo el $\mathrm{Cl}^{-}(\mathbf{9 5 \%})$ procede del mar, que 
$\operatorname{Ca}(\mu$ eq / $)$

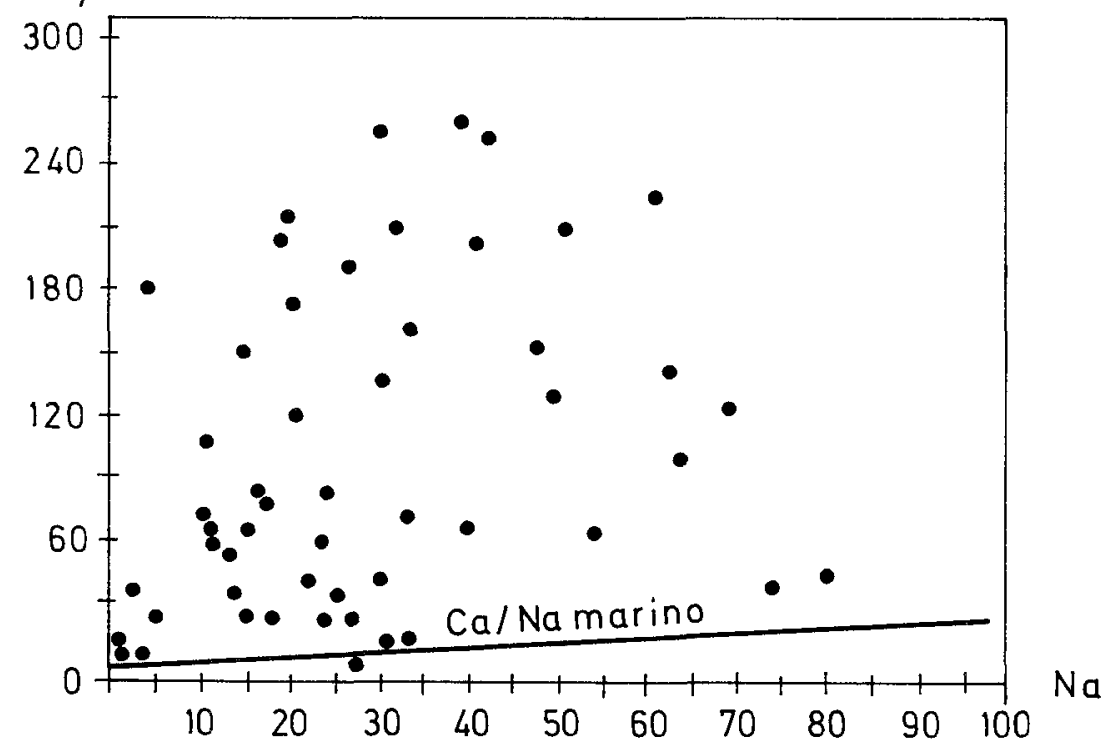

$\mathrm{SO}_{4}(\mu \mathrm{eq} / 1)$

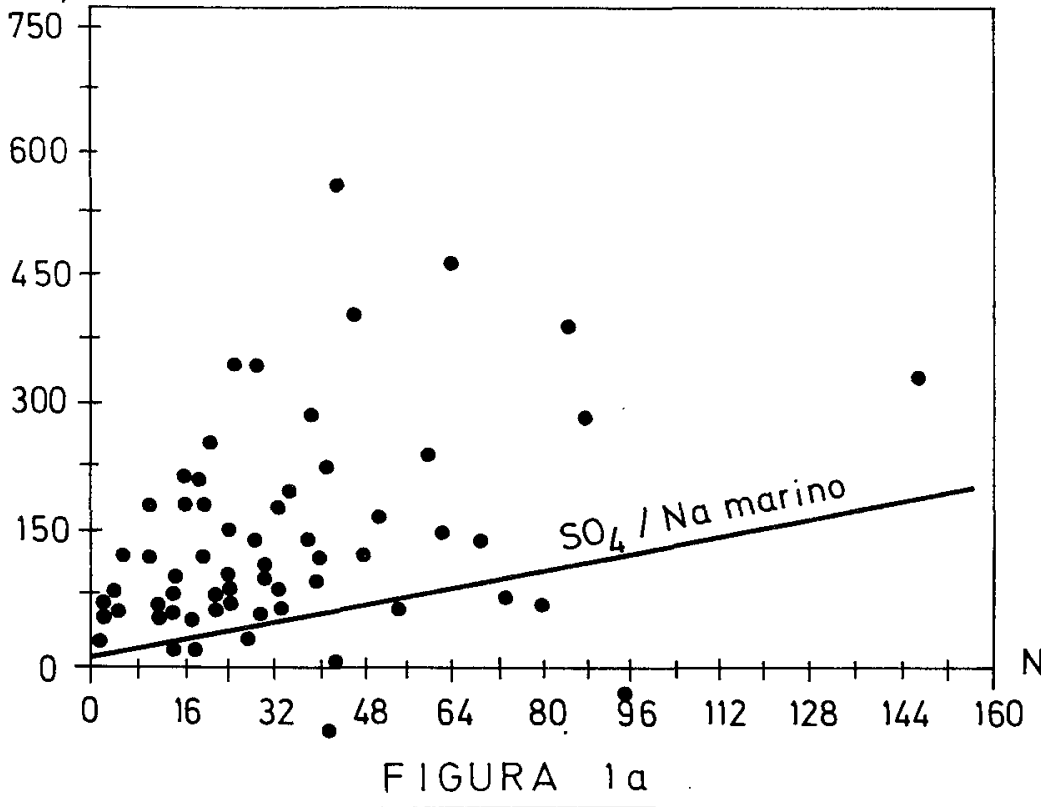

Figura 1 (a, b, c).-Concentraciones $(\mu \mathrm{eq} / 1)$ de $\mathrm{Ca}^{2+}, \mathrm{Mg}^{2+}, \mathrm{SO}_{4}^{2+}, \mathrm{K}+\mathrm{y} \mathrm{Cl}$, representadas respecto a la de $\mathrm{Na}^{+}$en la deposición global en cada una de las precipitaciones recolectadas. En trazo continuo aparece la relación entre cada par de iones correspondiente al agua del mar. 

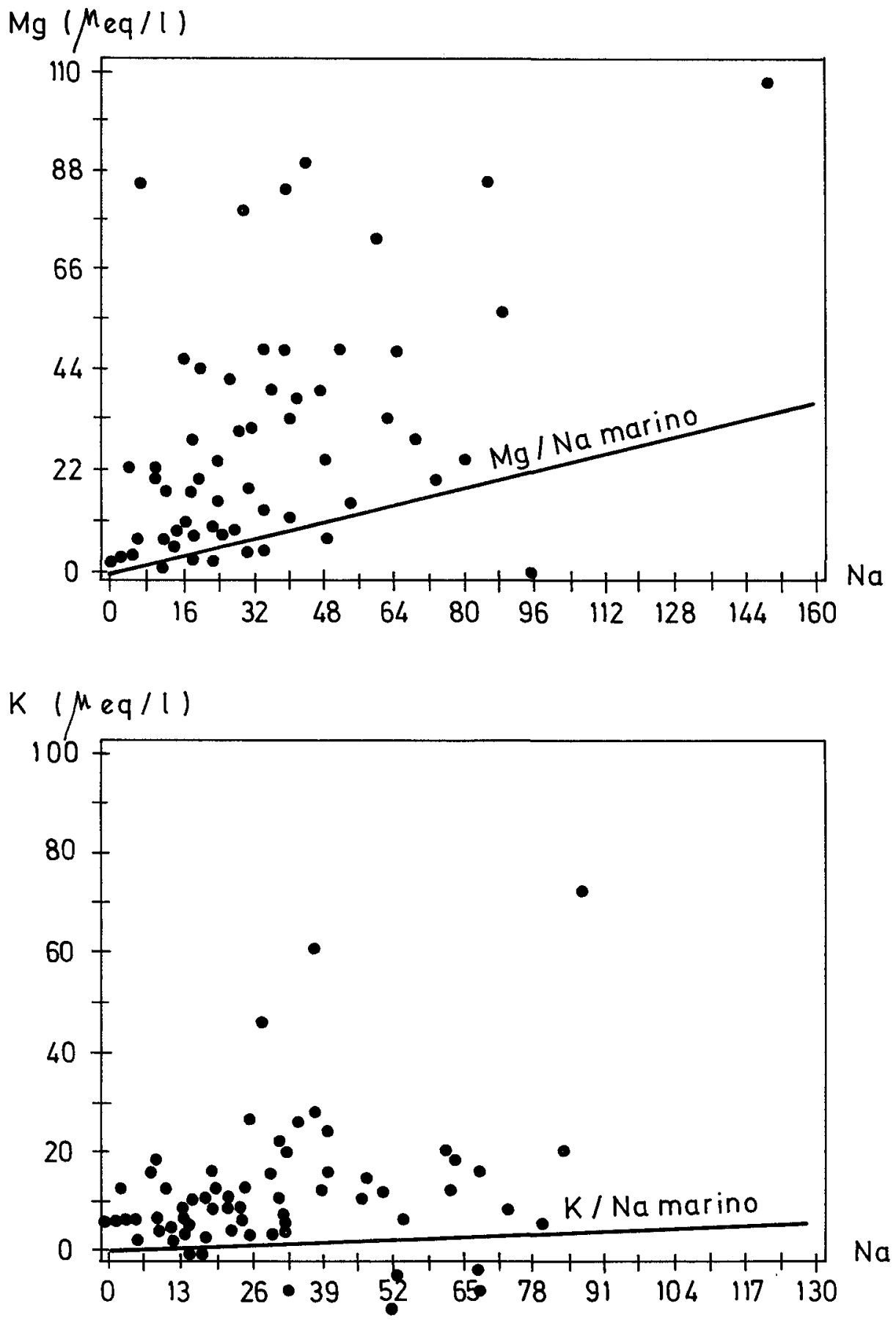

FIGURA Ib 


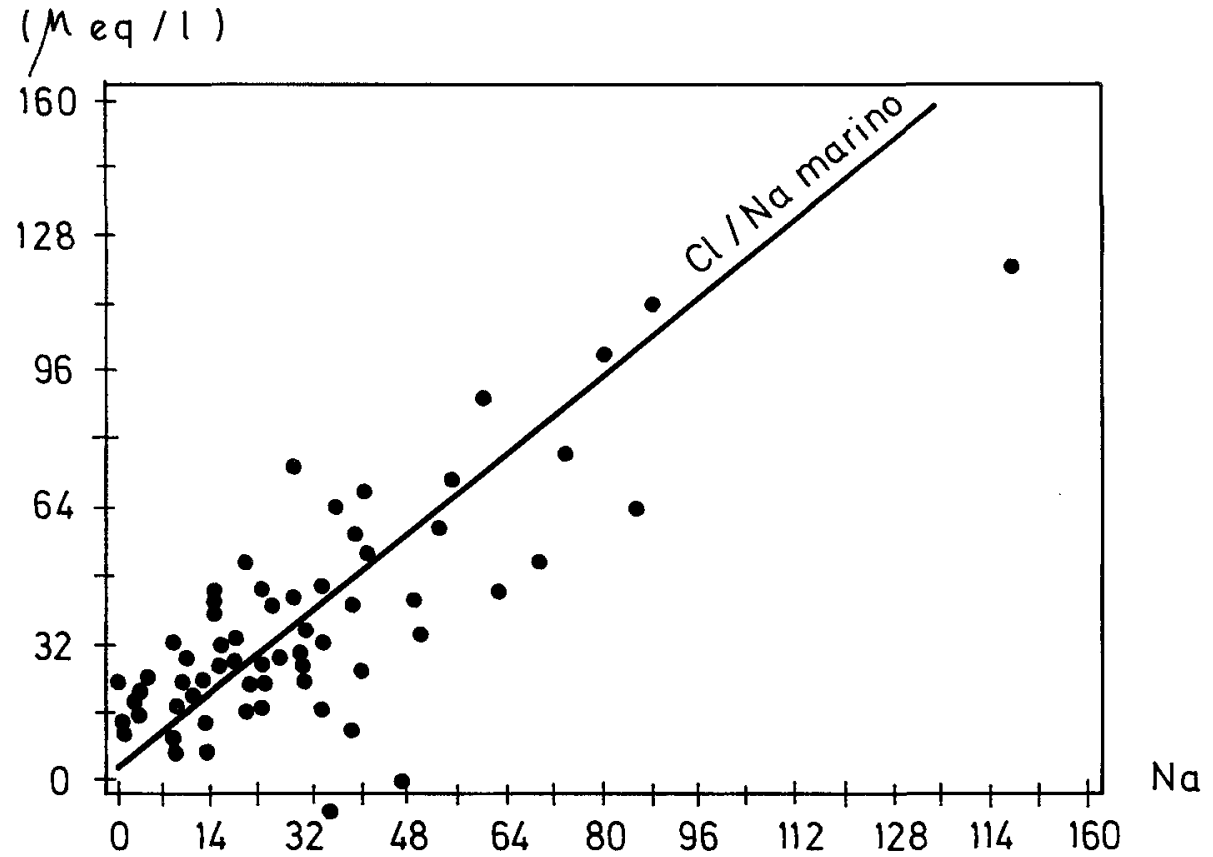

FIGURA IC

la mitad del $\mathrm{Mg}^{2+}$ tiene la misma procedencia, que sólo es atribuíble a esta fuente el $14 \%$ del $\mathrm{K}^{+}$y menos del $10 \%$ del S-SO ${ }_{4}^{2-}$, en tanto que del $\mathrm{Ca}^{2+}$ sólo tiene este origen un $2 \%$ del total. Aunque con tasas muy diferentes, SCHLESINGER et al. (1982), atribuyen al mar porcentajes similares de los iones analizados en las precipitaciones de Santa Ynez Mountains (California).

\section{Análisis de la acidez de las precipitaciones}

La precipitación ácida $(\mathrm{pH}<4.5)$ es un conocido fenómeno que afecta a extensas regiones de Europa y Norte América. Sus causas se deben al aumento del $\mathrm{SO}_{4} \mathrm{H}_{2}, \mathrm{NO}_{3} \mathrm{H}$ y ClH en la atmósfera, que se originan por precursores emitidos en una amplia variedad de actividades humanas (GALLOWAY, 1979). Las primeras referencias al tema, datan de los años 50 para Europa, (GORHAM, 1955) y de los 60 para Norte América (FISHER et al., 1968) y hasta ahora a pesar de las fluctuaciones temporales y regionales, el problema persiste en múltiples localidades.

En España, son escasos los datos acerca de la acidez de las precipitaciones. En 1978, Gracia y Elejalde, estudian el problema, circunscribiéndolo a 


\begin{tabular}{lcccccc}
\hline & $\mathrm{Na}$ & $\mathrm{Cl}$ & $\mathrm{Mg}$ & $\mathrm{K}$ & $\mathrm{S}^{-\mathrm{SO}_{4}}$ & $\mathrm{Ca}$ \\
\hline L'AVIC $\ldots \ldots \ldots .$. & 100 & 95.1 & 56.1 & 14.3 & 9.5 & 2.0 \\
MONTSENY .. & 100 & 95.5 & 42.5 & 13.5 & 6.0 & 1.7 \\
\hline
\end{tabular}

Tabla 4.-Porcentajes de los iones presentes en la precipitación atribuibles a origen marino, si se acepta como referencia el $100 \%$ de $\mathrm{Na}$ marino.

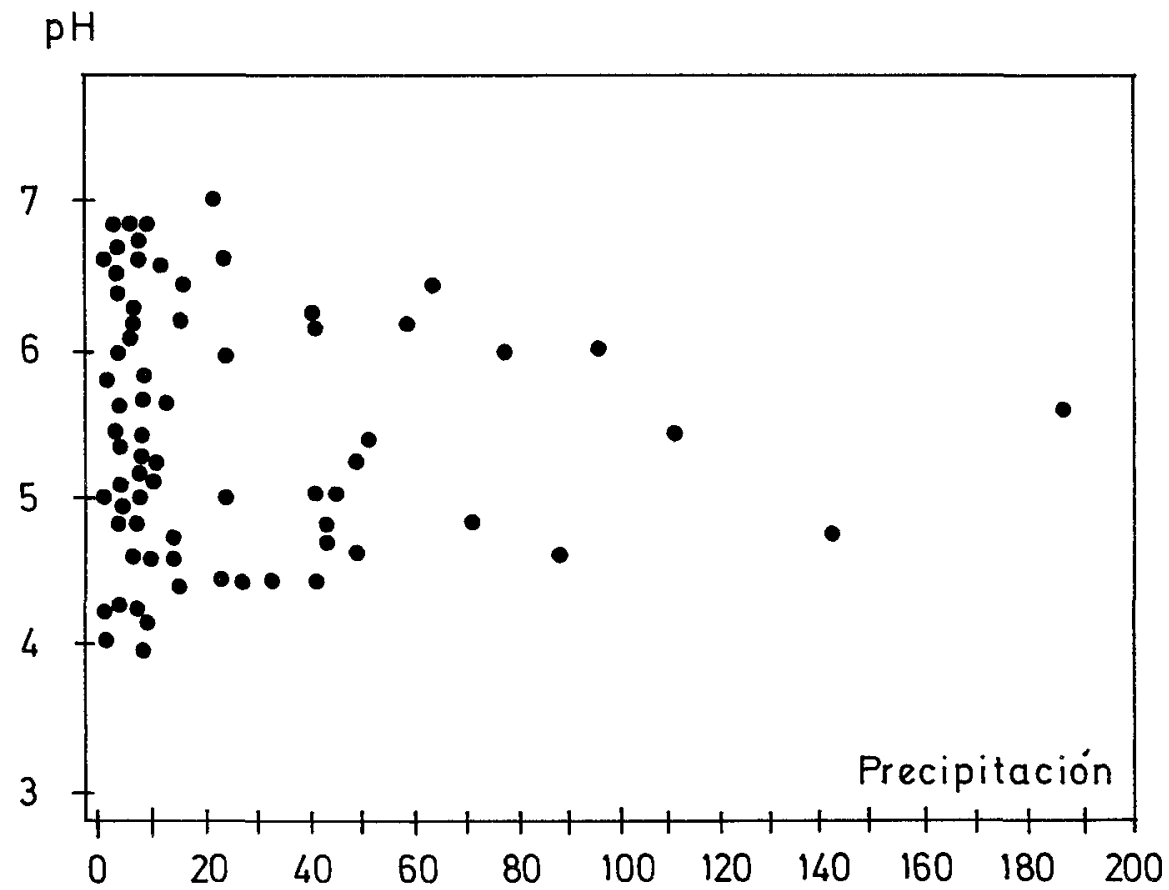

Figura 2.-Valores del pH del agua de lluvia en función del volumen de precipitación.

las áreas urbanas de Bilbao y Barcelona. Más adelante, se detectan lluvias ácidas en zonas naturales (ESCARRÉ et al., 1982; RODÁ, 1983). En l'Avic, en tres años de estudio, el $\mathrm{pH}$ medio es de 4.96 , por lo que la precipitación en la zona debe considerarse como moderadamente ácida.

Frecuentemente se ha encontrado una relación directa entre la acidez y volumen de precipitación. En L'Avic esta correlación negativa no es tan evidente. En la fig. 2, se muestra que es clara la gran dispersión en los valores 


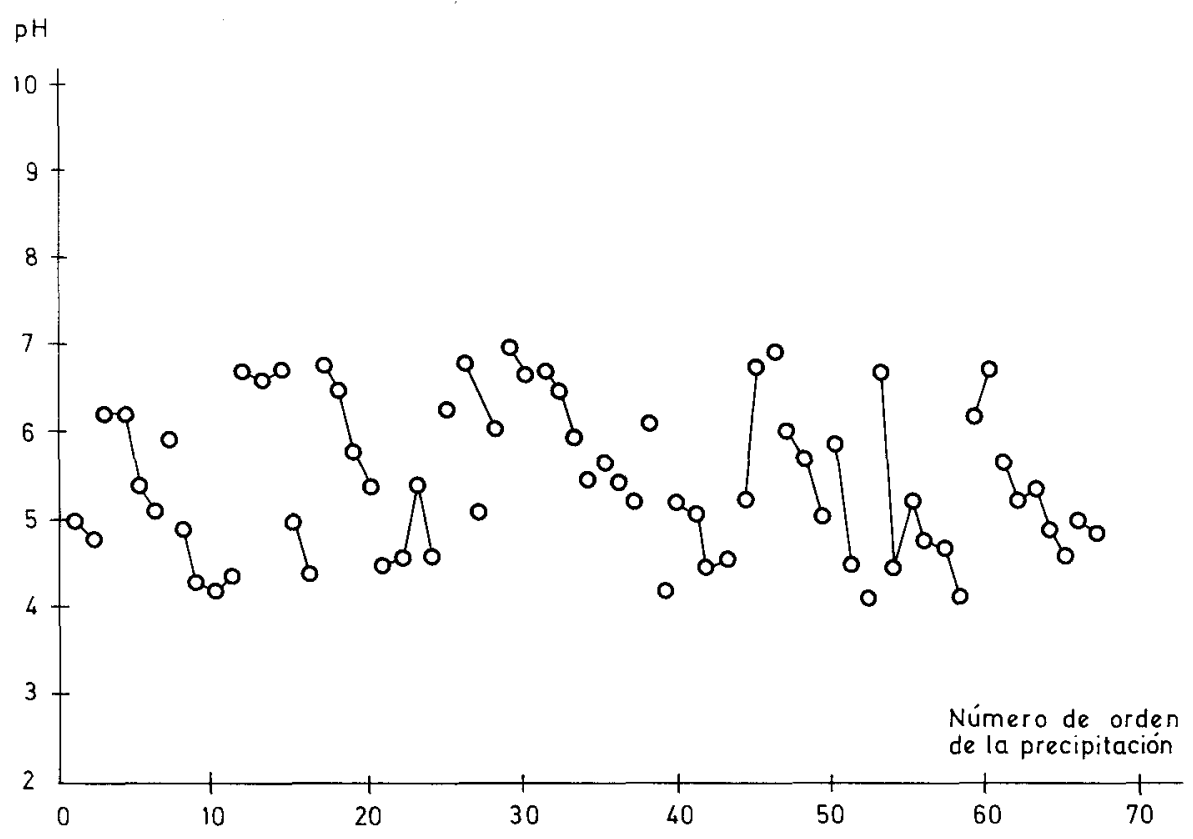

Figura 3.- Variaciones del pH de la deposición global en las muestras ordenadas cronológicamente y que aparecen separadas en el eje horizontal por intervalos regulares. Se han unido por trazos continuos todas las series de lluvias producidas en intervalos no mayores de 48 horas.

de $\mathrm{pH}$ con lluvias de menos de $20 \mathrm{~mm}$. En tanto que las más cuantiosas tienden a estabilizar el $\mathrm{pH}$ entre 4.5 y 6 . El valor más bajo de $\mathrm{pH}$ registrado fue de 3.9, en una precipitación de $0.5 \mathrm{~mm}$., en tanto que el más básico $(\mathrm{pH}=7.4)$ correspondió a una precipitación de $4 \mathrm{~mm}$. Posteriormente se han medido valores de $\mathrm{pH}$ de hasta 8 (Rovira y Piñol, com. verbal).

Si la cantidad de precipitación no es un claro condicionante del pH de la lluvia ( $\mathrm{r}=-0.089$, n.s.), en cambio sí es posible observar un cierto descenso de $\mathrm{pH}$ cuando se controlan series de lluvias sucesivas en cortos períodos de tiempo. En la fig. 3, se ha representado el valor de $\mathrm{pH}$ en cada día de precipitaçión. Se unen con líneas aquellas lluvias producidas a intervalos de horas o de como máximo 1 ó 2 días, quizás procedentes de un mismo frente desestabilizador. Aunque se observan bastantes puntos aislados, en el $84 \%$ de las agrupaciones efectuadas se aprecia que el pH disminuye desde la primera a la última precipitación. La interpretación más plausible sería atribuir esta disminución del $\mathrm{pH}$, al lavado que se efectúa en la baja atmósfera por las primeras lluvias caídas. Éstas arrastrarían las sales neutralizantes acumuladas allí (washout), y posteriormente la lluvia tendría la acidez propia del agua de la alta atmósfera (rainout), por lo general más ácida (HENIKSEN, 1979; FOWLER, 1980). También podría deberse en parte a que la deposición seca, de este tipo de sales, sobre los colectores es menos importante cuando las precipitaciones se repiten en un corto intervalo de tiempo. 
La importancia de la acidez en una zona como l'Avic se puede valorar en base a lo que representan las lluvias ácidas frente al volumen anual del agua caído en la zona. Una distribución de todas las lluvias según $\mathrm{pH}$ y volumen dió como resultado que sólo el $7.50 \%$ del volumen anual tiene $\mathrm{pH}<4.5$, presentando la mayor parte del agua $(48 \%)$ un $\mathrm{pH}$ entre 4.5 y 5.5 . El resto de agua tiene pH entre 5.5 y $7(42 \%)$, en tanto que por encima de 7 , sólo se da en el $2 \%$ restante.

Esta distribución de acidez en la lluvia, es muy similar a la detectada por RODÁ (1983) en Montseny. Sin embargo, es muy diferente a la encontrada en dos zonas de ámbito más agrícola, la Violada, SW de la provincia de Huesca, (Aguinaco, com. verb.) y Albatera, S de la provincia Alicante, (Sánchez y Chirino com. verb.) En estas últimas, dominan con claridad las lluvias no ácidas, con el $100 \%$ de los valores de $\mathrm{pH}$ por encima del $5.5 \mathrm{y}$ el $50 \%$ por encima de 7, con pH medio de 6.82 para La Violada y 6.42 para Albatera. Este comportamiento tan diferente podría corresponder a zonas de mayor aridez, con escasa vegetación y con mucha actividad agrícola, donde se producen frecuentes remociones de tierra que generan la incorporación, a las capas más bajas de la atmósfera, de polvo con capacidad neutralizante.

GORHAM et al. (1984) y GRANAT (1972) proponen un sencillo sistema para averiguar qué porcentaje de $\mathrm{H}^{+}$presente, está justificado por cada uno de los iones $\mathrm{SO}_{4}^{2-}, \mathrm{NO}_{3}^{-}$y alcalinidad. Según este modelo y por medio de regresiones múltiples, se ha calculado que sólo el $10 \%$ de los $\mathrm{H}^{+}$estaban asociados al $\mathrm{SO}_{4}^{2-}$, que el $24 \%$ lo están al $\mathrm{NO}_{3}^{-}$, y el $28 \%$ a la alcalinidad. En total estas tres posibles fuentes de acidez, sólo capturan en L'Avic algo más de la mitad de los hidrogeniones presentes en el agua de lluvia. De todas formas, la fiabilidad de estos porcentajes debe tomarse con precaución, ya que el modelo supone que las variables no están relacionadas entre sí, lo que no es cierto al tratarse de agua de precipitación. El $39 \%$ de los $\mathrm{H}^{+}$presentes en L'Avic deben atribuirse a otras fuentes. GALLOWAY et al. (1976) definen diversas substancias químicas que pueden actuar como creadoras de acidez en la lluvia (ácidos débiles y ácidos Bronsted) al margen de la acidez libre debida a los ácidos fuertes (sulfúrico y nítrico).

\section{Correlaciones iónicas en el agua de precipitación}

La naturaleza de las relaciones entre los iones presentes en la precipitación de l'Avic, se ha investigado de dos maneras: mediante regresiones lineales, y con una aproximación multivariante. GORHAM et al. (1984) y ALTWICKER et al. (1986) ponen en evidencia la idoneidad de estas técnicas de análisis estadístico para determinar el grado de conexión mutua entre los iones de la lluvia.

En la tabla 5, se muestra la matriz de coeficientes de correlación lineal, calculados entre todos los iones analizados, conductividad y volumen de precipitación con todos los datos y sin previa agrupación por fechas o tipos de Iluvias. Destaca el gran número de correlaciones significativas existentes para un nivel de probabilidad del $95 \%$. $\mathrm{El} \mathrm{H}^{+}$y alcalinidad son los iones que presentan menor número de correlaciones significativas. 


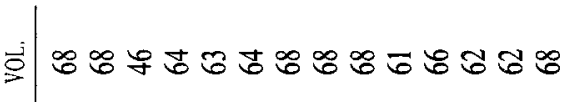

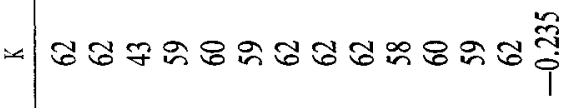

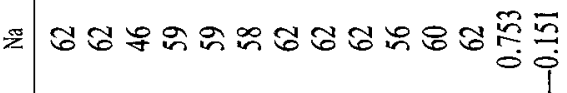

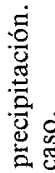

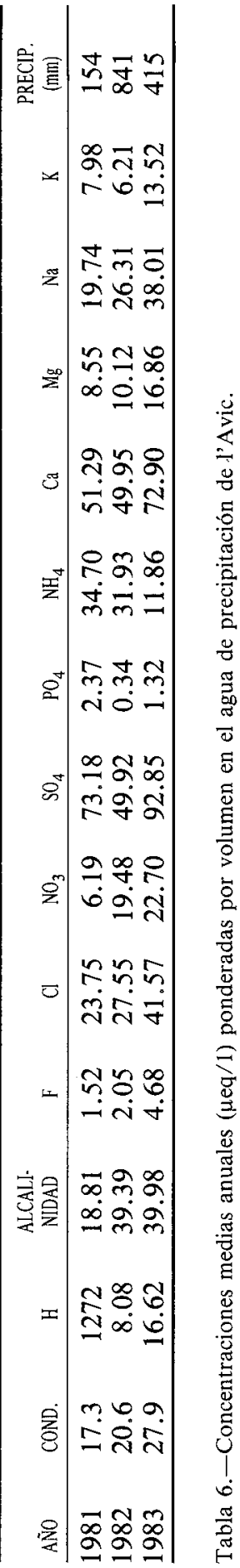

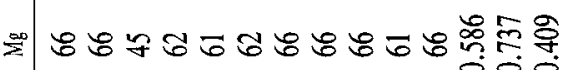

త

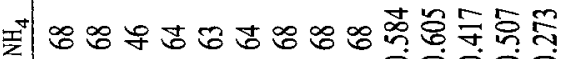
0ㅇㅇㅇㅇ

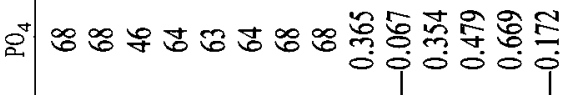

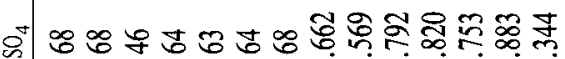
융ㅇㅇㅇㅇㅇㅇㅇ

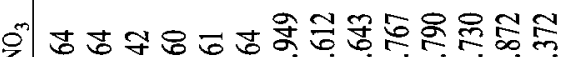

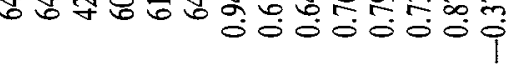

ஏ

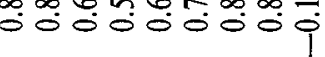

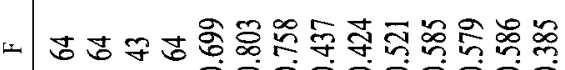

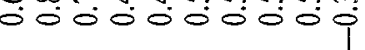

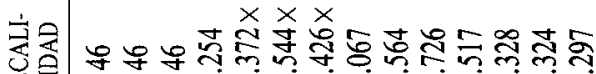

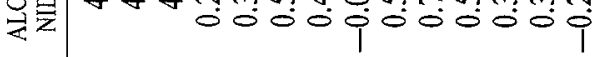

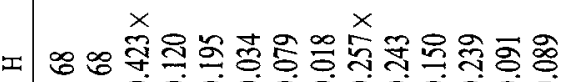

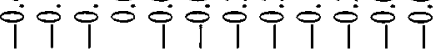

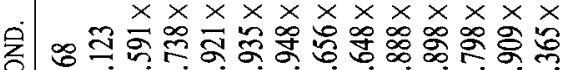

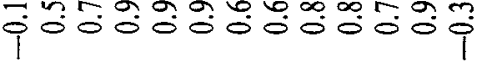


El volumen de lluvia afecta negativamente a la concentración de todos los iones excepto $\mathrm{Cl}^{-}$y $\mathrm{Na}^{+}$. El origen marino de ambos y el predominio de lluvias procedentes del levante justifica este hecho.

La emisión de sustancias a la atmósfera se hace en mezclas de compuestos, de los que en muchas ocasiones las proporciones son muy constantes. Es por esto que determinadas relaciones iónicas que se observan en el agua de lluvia por métodos de análisis multivariante, pueden atribuirse a la procedencia común de los iones. De esta forma GORHAM et al. (1984) consiguen reflejar por medio de una clasificación multivariante que los tres factores que determinan el quimismo de la precipitación en el este de los EE. UU. son la contaminación de aire, la agricultura y sprays marinos.

En L'Avic, los resultados de un análisis multivariante de componentes principales, aparecen en la fig. 4. En ella se observa la situación de 10 iones del agua de lluvia en el espacio reducido a los tres primeros ejes, que absorben más del $65 \%$ de la varianza total. El eje I representa el grado de concentración de las lluvias, mientras que el II parece recoger la influencia marina y acidez, ambas claramente opuestas.

\section{Variación temporal de la química de la precipitación}

La concentración media mensual de la mayoría de los iones muestra una cierta pauta estacional (fig. 5). Todos excepto $\mathrm{Cl}^{-}$y Na $\mathrm{Na}^{+}$, aparecen con máximos más o menos patentes en el verano y con tendencia a tener menores concentraciones en invierno y otoño. En el $\mathrm{Cl}^{-}$y $\mathrm{Na}^{+}$, al margen de los grandes valores de julio, se observa una tendencia a máximos invernales. La pauta reseñada no parece que se pueda atribuir a la contaminación atmosférica o agrícola, como ocurre en otras zonas (SWANK y HENDERSON, 1976; ROSEN, 1982; GRANAT, 1978). El ritmo de precipitaciones en la zona mediterránea con mínimos estivales, y la intensa actividad agrícola en estos meses pueden ser las causas de estos máximos de verano. El predominio de $\mathrm{Cl}^{-}$y $\mathrm{Na}^{+}$en primavera y otoño pudiera deberse a la abundancia de lluvia de origen marino, dada la frecuencia de vientos de levante en dichas épocas (TERRADAS y ESCARRE, 1983).

La variabilidad intermensual se comprobó mediante un análisis de la varianza. El resultado indica que el factor temporal es significativo para los iones $\mathrm{NO}_{3}^{-}, \mathrm{PO}_{4}^{3-}, \mathrm{Ca}^{2+}, \mathrm{Mg}^{2+}, \mathrm{Na}^{+}$y los hidrogeniones. Para el resto $\left(\mathrm{K}^{+}, \mathrm{NH}_{4}^{+}\right.$, $\mathrm{SO}_{4}^{2-}, \mathrm{Cl}^{-}, \mathrm{F}^{-}$y alcalinidad) las diferencias mensuales en la concentración carecían de significación.

A escala anual, los valores medios, ponderados por volumen, para los 3 años de estudios (en 1981 sólo 5 meses), indican una enorme variabilidad en todos los iones (tabla 6). Excepto para el $\mathrm{NH}_{4}^{+}, 1983$ es el año con concentraciones más elevadas, que llegan a doblar en diversos iones la concentración media de éstos para 1981. Sólo el $\mathrm{Ca}^{2+}$ presenta diferencias moderadas entre uno y otro año. Sin embargo, a pesar de las diferencias interanuales, observamos que los iones dominantes son siempre $\mathrm{SO}_{4}^{2-}, \mathrm{Ca}^{2+}, \mathrm{Cl}^{-}$y alcalinidad. 
$50.8 \%$

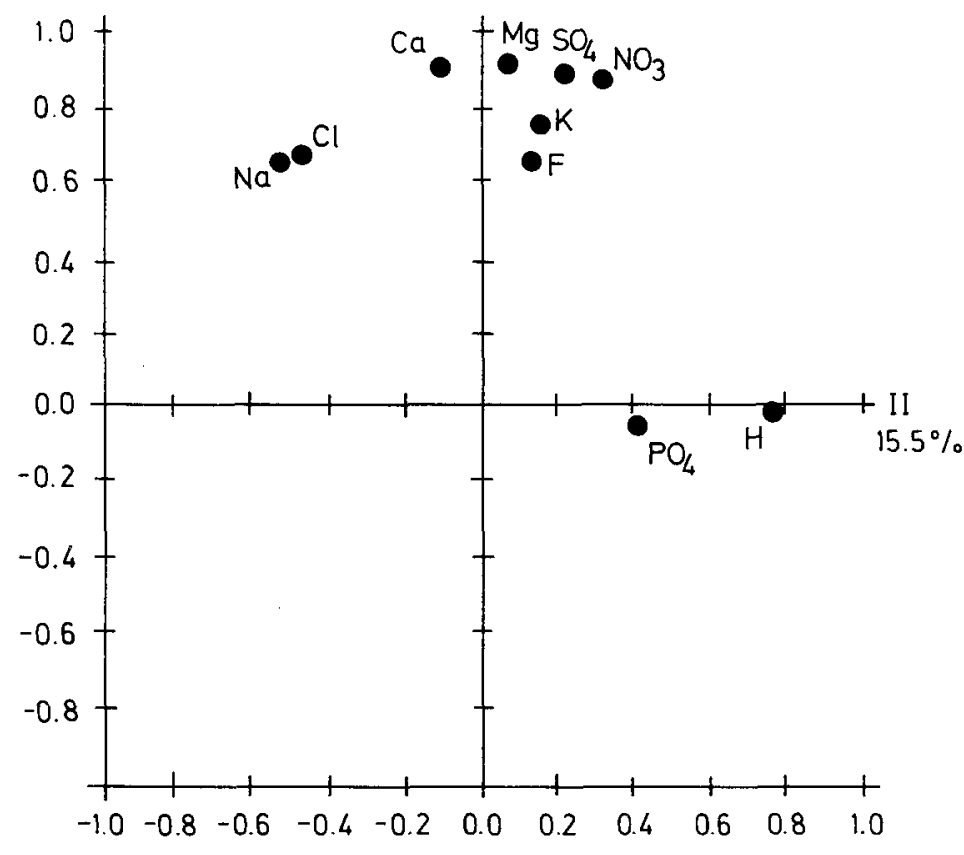

Figura 4.-Localización de los iones de la deposición global en el espacio definido por los ejes I y II de un análisis de componentes principales realizado con sus concentraciones en $\mu \mathrm{eq} / 1$.

\section{Aportes anuales por precipitación}

La gran variabilidad interanual observada a nivel de concentraciones, no se aprecia tan claramente cuando comparamos los aportes anuales. En la tabla 7 , se muestran dichos aportes para los dos años completos estudiados, y las entradas anuales para $583 \mathrm{~mm}$. de precipitación media.

La diferencia entre la cantidad de precipitación de los dos años, hace que en los aportes se reduzcan las diferencias apreciadas en las concentraciones, e incluso que se invierta el orden de los años según la magnitud de las entradas.

Destacan por su constancia interanual los aportes de $\mathrm{K}^{+}, \mathrm{Mg}^{2+}, \mathrm{F}^{-}$y los hidrogeniones. En cambio $\mathrm{NO}_{3}^{-}$y $\mathrm{Na}^{+}$presentan diferencias en el primer año que casi igualan el valor de 1983. El resto de iones presentan diferencias más moderadas, aunque siempre con valores mayores para 1982. Los aportes anuales de $\mathrm{S}_{-} \mathrm{SO}_{4}^{2-}, \mathrm{Ca}^{2+}, \mathrm{Cl}^{-}$y NO $\mathrm{NO}_{3}^{-}$son semejantes (tabla 7), y varían entre $6.3 \mathrm{y}$ $6.8 \mathrm{Kg} . /$ ha. $\mathrm{El} \mathrm{Na}{ }^{+}, \mathrm{NH}_{4}^{+}$y K${ }^{+}$entran en cantidades moderadas $(3.8,2.8 \mathrm{y}$ $1.9 \mathrm{~kg} / \mathrm{ha}$, respectivamente), en tanto que $\mathrm{P}_{-} \mathrm{PO}_{4}^{3-}, \mathrm{F}^{-}$y $\mathrm{Mg}^{2+}$ no alcanzan $1 \mathrm{Kg} / \mathrm{ha} /$ año. 

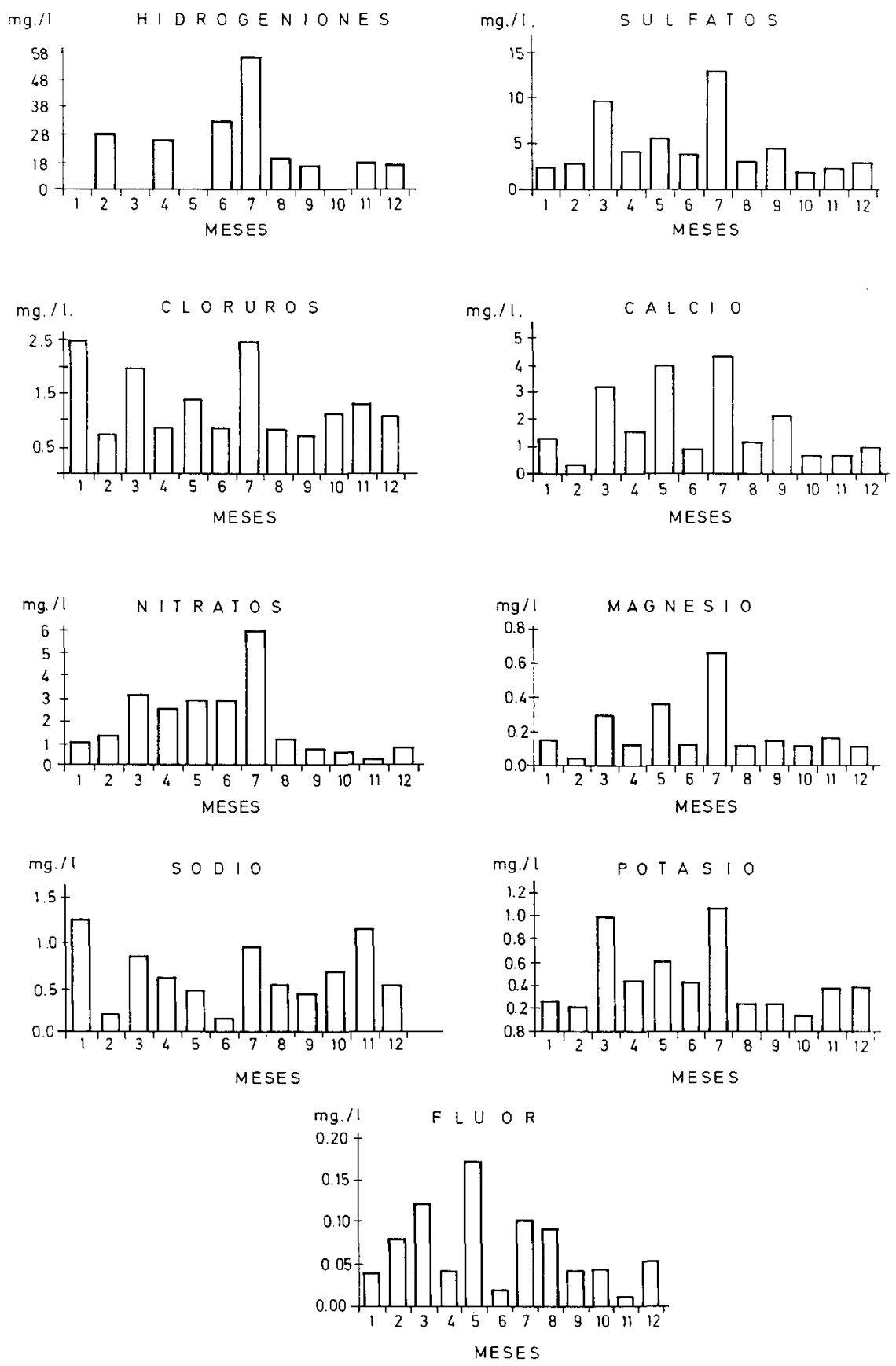

Figura 5.-Variaciones medias mensuales de las concentraciones en $\mathrm{mg} / 1$ de los principales iones analizados en la deposición global de l'Avic. 
Se aprecia que la magnitud de las entradas es similar a las de otras zonas (tabla 8). Observamos mayor similitud entre l'Avic y Montseny (RODÁ, 1983), que entre éstas y el resto. A pesar de que las estaciones de Montseny, Violada y Albatera, están situadas también en el área mediterránea, las dos últimas se sitúan en zonas cuyos alrededores desarrollan una intensa actividad agrícola. Las mayores diferencias entre las dos estaciones de montaña Prades y Montseny, se deben a los importantes aportes recibidos por el Montseny en $\mathrm{NO}_{3}^{-}$ y $\mathrm{Ca}^{2+}(10 \mathrm{~kg} / \mathrm{ha})$, en tanto que el resto son más parecidos.

Las dos zonas agrícolas presentadas, Violada y Albatera (Sánchez y Chirino, com. verbal; Aguinaco, com. verbal) presentan algunas características comunes que las diferencian del resto, especialmente sus reducidos aportes en iones $\mathrm{H}^{+}$y los relativamente altos aportes de iones en proporción a la baja precipitación.

La estación de Galicia (CALVO DE ANTA et al., 1979) manifiesta las características de una zona natural, antropizada y con mucha influencia marina. Las entradas anuales de $\mathrm{NO}_{3}^{-}$, que se citan son sorprendentemente elevadas, aunque podrían ser justificables por la existencia de alguna fuente industrial en las proximidades.

Como referencia de zonas más alejadas, se citan los datos de dos sistemas naturales: los robledales húngaros (SZABÓ, 1985), y la estación experimental de Hubbard Brook (USA) (LIKENS et al. 1977). La comparación con los datos de Hungría es más clara, dada la similar precipitación de dicha zona con las españolas. La falta de datos en $\mathrm{Na}^{+}$, no permite evaluar la influencia marina, ya que los considerables aportes de $\mathrm{Cl}^{-}$, pueden tener en parte origen continental (LIKENS et al., 1977; SCHLESINGER et al., 1982). A juzgar por los aportes en $\mathrm{Ca}^{2+}, \mathrm{NO}_{3}^{-}$y sobre todo en $\mathrm{S}_{-} \mathrm{SO}_{4}^{2-}$, se constata la fuerte influencia continental en la química de la precipitación en esta zona del norte de Hungría.

Los aportes por precipitación en Hubbard Brook son claramente diferentes al resto de la tabla 8: la mayor acidez de la lluvia, junto a los grandes aportes de $\mathrm{NO}_{3}^{-}$y $\mathrm{SO}_{4}^{2-}$, responsables de la misma (GALLOWAY, 1979; LIKENS et al. 1977), son sus características más sobresalientes.

\section{Importancia de los aportes atmosféricos para el encinar}

Es frecuente comparar la magnitud de los aportes atmosféricos con la incorporación anual de los mismos que se produce en la vegetación perenne. SWANK (1984) indica que en Walker Branch y Coweeta, el nitrógeno de la precipitación global representa el $70 \%$ del incorporado anualmente por la biomasa aérea, en tanto que las entradas de $\mathrm{Ca}^{2+}$ y K${ }^{+}$, suponen entre el 20 y $40 \%$ del incorporado, según la zona. Sin embargo, como indica este autor las estimas atmosféricas por deposición global son subestimas ya que la deposición de gases y aerosoles no se suele cuantificar.

En L'Avic el nitrógeno se analizó en dos de sus tres formas más frecuentes, $\mathrm{NO}_{3}^{-}$y $\mathrm{NH}_{4}^{+}$, sin embargo el $\mathrm{N}$ orgánico no se estimó. MATZNER et al. (1982) calcula que en Solling las entradas de $\mathrm{N}$ total son de $24.7 \mathrm{~kg} / \mathrm{ha} /$ año, de los que $4.8 \mathrm{~kg} / \mathrm{ha}$ corresponden al $\mathrm{N}$ orgánico. $\mathrm{Si}$ comparamos las proporciones dadas por estos autores, a las formas de nitrógeno estimadas en l'Avic 


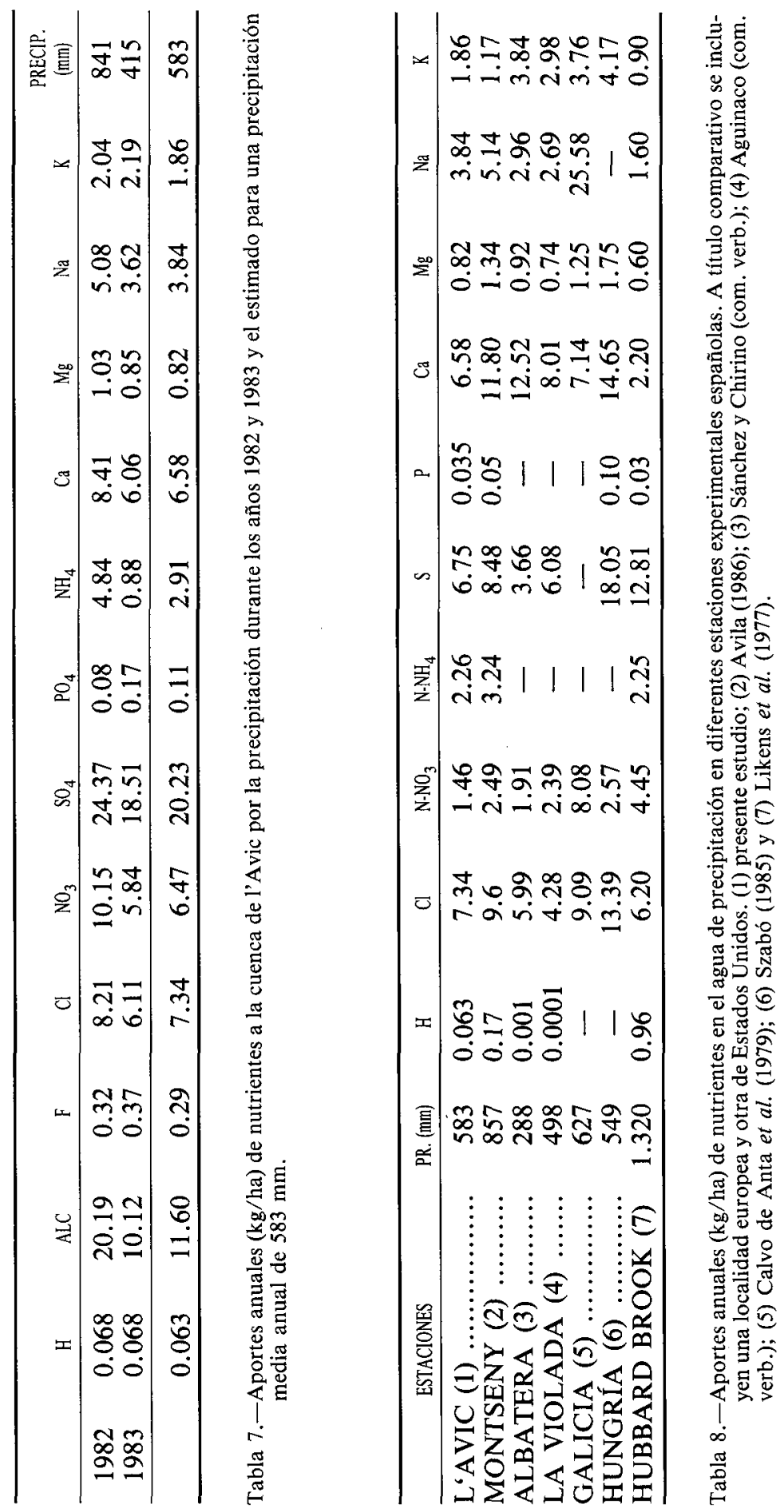


observamos una gran coincidencia, por lo que no sería muy erróneo evaluar la cantidad de $\mathrm{N}$ orgánico esperable, si se mantienen las proporciones relativas. De este modo se estimaría una entrada adicional de $1 \mathrm{~kg} / \mathrm{ha} / \mathrm{año}$, lo que elevaría los aportes de $\mathrm{N}$ total hasta $5 \mathrm{~kg} / \mathrm{ha} /$ año.

ÁVILA (1986) indica que los $6 \mathrm{~kg} / \mathrm{ha} /$ año recibidos por deposición global en el Montseny, suponen el 33\% de $\mathrm{N}$ acumulado anualmente en la biomasa aérea del encinar. Si se compara con los aportes para l'Avic, y se supone una producción similar para este encinar, probablemente los aportes atmosféricos de $\mathrm{N}$, no alcanzarían ni el $\mathbf{2 0 \%}$ de los acumulados anualmente.

Desde la óptica de la gestión ambiental de los bosques, los aportes atmosféricos por deposición son importantes dada la falta de fertilización como práctica forestal. Como indica SWANK (1984), la extracción total o parcial de los árboles talados y la frecuencia de las talas se podrían regular mejor si se conociesen con mayor precisión los flujos de nutrientes en los ecosistemas, y en particular los aportes externos de aquellos nutrientes escasos o limitantes para los mismos.

\section{AGRADECIMIENTOS}

Los autores queremos dejar constancia de nuestro agradecimiento a doña María José Lledó por su importante participación en el proyecto de investigación en que se incluye el presente estudio. En segundo lugar damos las gracias a don Juan Rafael Sánchez, don Esteban Chirino y doña Maite Aguinaco por habernos proporcionado datos inéditos de sus zonas de estudio. A los profesores Jaume Terradas y Ferrán Roda les quedamos reconocidos por haber realizado una revisión del manuscrito original y por las valiosas sugerencias aportadas al mismo.

Para la realización de esta investigación se ha contado con medios económicos del Proyecto Cooperativo Hispano-Norteamericano $n .{ }^{\circ} 370$, con la ayuda de CAICYT n. ${ }^{\circ} 770$ y una financiación adicional del proyecto LUCDEME. 
ALTWICKER, E. R.; P. E. SHANAGHAN \& A. H. JOHANNES 1986: Atmospheric deposition to remote receptors. III. Statistical analysis of precipitation data from the ILWASNETWORK. Water, air and soil pollution, 28: 71-88.

AVILA, A. 1986: Composición química de la lluvia en el Montseny, en: Bases ecológicas para la gestión del medio ambiente. Montesquieu, Diputación de Barcelona.

BATES, T. S.; R. J. CHARLSON \& R. H. GAMMON 1987: Evidence for the climatic role of marine biogenic sulphur. Nature 329: 319-321.

CALVO DE ANTA, P. GONZÁLEZ \& J. DÍAZ 1979: Nuevos datos sobre la influencia de la vegetación en la formación del suelo en Galicia. II. Aportes de elementos por lavado de cubierta y tronco. Anal. Edaf. y Agrobiología 1675-1691.

CHARLSON, R. J.; J. E. LOVELOCK; M. O. ANDREAE \& S. G. WARREN 1987: Oceanic phytoplankton, atmospheric sulphur, cloud albedo and climate. Nature 326: 655-661.

EATON, J. S.; G. E. LIKENS \& F. H. BORMANN 1980: Wet and dry deposition of sulphur at Hubbard Brook, in Hutchinson \& Havas (eds.) Effects of acid precipitation on terrestrial ecosystems. Plenum Publ. Co. pp. 69-75.

ESCARRE, A.; J. BELLOT; M. J. LLEDÓ; J. HERVAS; E. PUJALTE \& P. GARRIDO 1982: Balance de nutrientes en pequeñas cuencas de encinar. I. Selección de la zona de estudio e instalación de instrumentos para la recogida de datos. Mediterranea, Ser. Biol. 6: 141-165.

ESCARRE, A.; J. BELLOT; M. J. LLEDÓ; A. CLEMENTE; J. R. SÁNCHEZ \& A. ESCLAPES 1983: Nutrient budgets in a forested mediterranean watershed. MAB-5 Workshop, Budapest.

ESCARRE, A.; M. J. LLEDÓ; J. R. SÁNCHEZ; A. CLEMENTE; J. BELLOT; A. ESCLAPES \& A. ROVIRA 1984: Compartimentos y flujos biogeoquímicos en un encinar, distinto predominio de factores físicos y biológicos en su control. Revista del Jardín Botánico Nacional, La Habana, vol. V, 13: 65-80.

ESCARRE, A.; J. BELLOT; M. J. LLEDÓ; J. MARTÍN; A. ESCLÁPES; E. SEVA; A. ROVIRA \& J. R. SÁNCHEZ 1986: Balance hídrico, meteorización y erosión en una pequeña cuenca de encinar mediterráneo. Monografías ICONA 47: 54-115.

ESCARRE, A.; L. FERRES; R. LÓPEZ; J. MARTÍN; F. RODA \& J. TERRADAS 1987: Nutrient use strategy by evergreen-oak (Quercus ilex ssp. ilex) in NE Spain, en J. D. Tenhunen et al. (eds.) Plant response to stress. NATO ASI Series vol. G 15, Springer-Verlag.

ESCUDERO A.; B. GARCÍA; J. M. GÓMEZ \& E. LUIS 1985: The nutrient cycling in Quercus rotundifolia and Quercus pyrenaica ecosystems («dehesas») of Spain. Oecol. Plant. 6: 73-86.

FERRES, L. 1984: Biomasa, producción y mineralomasa del encinar montano de La Castanya (Montseny). Tesis doctoral, Universidad Autónoma de Barcelona, Bellaterra.

FERRES, L.; F. RODA; A. M. C. VERDÚ \& J. TERRADAS 1984: Circulación de nutrientes en algunos ecosistemas forestales del Montseny. Mediterranea Ser. Biol. 7: 139-166.

FISHER, D. W. 1968: Annual variations in chemical composition of atmospheric precipitation in eastern North Carolina and Southeastern Virginia. Geochemistry of water. Geological survey water-supply, paper 1535-M, $21 \mathrm{pp}$.

FISHER, D. W.; A. W. GAMBELL; G. E. LIKENS \& F. H. BORMANN 1968: Atmospheric contributions to water quality of streams in the Hubbard Brook experimental forest, New Hampshire. Water Resources Research 4 (5): 1115-1126.

FOLCH, R. \& E. VELASCO 1974: Dades cartográfiques per a l'estudi de la vegetació de les Muntanyes de Prades. XVIII Asamblea intercomarcal d'estudiosos. L'Espluga de Francolí.

FOWLER, D. 1980: Removal of sulphur and nitrogen compounds from the atmosphere in rain and by dry deposition En International Conference on the ecological impact of acid precipitation, Norway, pp. 32.

GALLOWAY, J. N. 1979: Acid precipitation: spacial and temporal trends. Workshop on Acid Rain, ASCE/Boston, Massachusetts, April 2-6, $20 \mathrm{pp}$.

GALLOWAY, J. N. \& G. E. LIKENS 1976: Calibration of collection procedures for the determination of precipitation chemistry. Water, air and soil pollution, 6: 241-258.

- 1978: The collection of precipitation for chemical analysis. Tellus, 30: 71-82.

GALLOWAY, J. N.; G. E. LIKENS \& E. S. EDGERTON 1976: Hydrogen ion speciation in the acid precipitation of the northeastern United States, en Proceeding of the firts International Symposium on acid precipitation and the forest ecosystem. USDA, Technical Report NE-23: 383-396.

GALLOWAY, J. N. \& G. E. LIKENS 1978: The collection of precipitation for chemical analysis. Tellus 30: 71-82. 
GAMBELL, A. W. \& D. W. FISHER 1966: Chemical composition of rainfall eastern North Carolina and southeastern Virginia. Geochemistry of water. Geological survey water-supply, paper $1535-\mathrm{k}, 41 \mathrm{pp}$.

GORHAM, E. 1955: On the acidity and salinity of rain. Geochimica et Cosmochimica Acta 7: 231-239.

- 1958: The influence and importances of daily weather conditions in the supply of chloride, sulphate and other ions to fresh waters from atmospheric precipitation. Freshwater Biological Association, 241, B-679; 147-178.

GORHAM, E.; F. B. MARTIN \& J. T. LITZAU 1984: Acid rain: ionic correlations in the Eastern United States, 1980-1981. Science 225: 407-409.

GOSZ, J. R. 1980: Nutrient budgets studies for forest along an elevational gradient in New Mexico. Ecology 61: 515-521.

GRACIA, I. \& C. ELEJALDE 1978: Estimación de la contaminación atmosférica en el agua de lluvia.

GRANAT, L. 1972: On the relation between $\mathrm{pH}$ and the chemical composition in atmospheric precipitation. Report AC-18/UDC-551-510.4. Institute of meteorology. University of Stockholm, $29 \mathrm{pp}$.

- 1978: Sulphate in precipitation as observed by the european atmospheric chemistry network. Atmospheric Environment, 12: 413-424.

HENIKSEN, A. 1979: A simple approach for identifying and measuring acidification of freshwater. Nature 278: 542-545.

LAKHANI, K. H. \& H. G. MILLER 1980: Assessing the contribution of crown leaching to the element content of rainwater beneath trees, en Hutchinson \& Haves (eds.) Effect of acid precipitation of terrestrial ecosystems. Plenum Pub. Co.: 161-172.

LEMEE, G. 1974: Recherches sur les écosystèmes des reserves biologiques de la forêt de Fontainebleau. IV. Entrées d'éléments mineraux par les précipitations et transfert au sol par le pluviolessivage. Oecol. Plant 9 (3): 733-735.

LIKENS, G. E. (ed.) (1981): Some perspectives of the major biogeochemical cycles. SCOPE 17, J. Willey \& Sons, $175 \mathrm{pp}$.

LIKENS, G. E.; F. H. BORMANN; R. S. PIERCE; J. S. EATON \& N. M. JOHNSON (1977): Biogeochemistry of a forested ecosystem. Springer-Verlag, $146 \mathrm{pp}$.

LINDBERG, S. E.; G. E. LOVETT; D. D. RICHTER \& D. W. JOHNSON 1986: Atmospheric deposition and canopy interactions of major ions in a forest. Science 231: 141-145.

LLEDÓ, M. J. \& A. ESCARRE 1984: Datos preliminares de la exportación de materia disuelta y particulada en una cuenca de encinar. Cuadernos de Investigación Geográfica XI, 1 y 2 : $52-63$.

MATZNER, E.; P. K. KHANNA; K. J. MEIWES; M. LINDHEIM; J. PRENZEL \& B. ULRICH 1982: Elementflüsse in Waldökosystemen in Solling. Datendokumentation. Göttinger Bodenkundliche Berichte 71: 1-267.

MESZAROS, E. 1981: Atmospheric chemistry. Fundamental aspects. Studies in environmental science 11. Elsevier Scientific pub., $193 \cdot \mathrm{pp}$.

MILLER, H. G. \& J. D. MILLER 1980: Collection and retention of atmospheric pollutants by vegetation. Proc. Int. conf. ecol. impact acid. precip. Norway, SNSF. Project pp. 33-40.

PIÑOL, J.; M. J. LLEDÓ; J. BELLOT; A. ESCARRE \& J. TERRADAS (en prensa). Evapotranspiration estimation and runoff response of two Mediterranean forested watersheds. MEDECOS, Montpellier.

REYNOLDS, B. 1984: An assessment of the spatial variation in the chemical composition of bulk precipitation within and upland catchment. Water Resources Research 20 (6): 733-735.

RODA, F. 1983: Biogeoquímica de les aigues de pluja i de drenatje d'alguns ecosistemes forestals del Montseny. Tesi Doctoral, Universitat Autonoma de Barcelona, Bellaterra.

ROSEN, K. 1982: Supply, loss and distribution of nutrients in three coniferous forest watersheds in central Sweden. Sveriges Lantbruksuniversitat. Upssala, $71 \mathrm{pp}$.

SCHLESINGER, W. H. \& W. A. REINERS (1974): Deposition of water and cations on artificial foliar collectors in Fir Krumm-holz of New England Mountains. Ecology 55: 378-386.

SCHLESINGER, W. H. \& M. M. HASEY 1980: The nutrient content of precipitation of southern California. The American Midland Naturalist, 103: 114-122.

SCHLESINGER, W. H.; J. T. GRAY \& F. S. GILLIAM 1982: Atmosheric deposition in a chaparral ecosystem of southern California. Water Resources Research 18 (3): 623-629.

SWANK, W. T. 1984: Atmospheric contributions to forest nutrient cycling. American water resources association 20 (3): 313-321. 
SWANK, W. T. \& G. S. HENDERSON 1976: Atmospheric input of some cations and anions to forest ecosystems in North Carolina and Tennessee. Water Resources Research 12 (3): 541-546.

SZABO, M. 1977: Nutrient content of throughfall and stemflow water in an oak-forest (Quercetum petraea-cerris) ecosystem. Acta Agronomica Academia Scientiarum Hungaricae 26 (34): 241-258.

- 1985: Ecology of an oak-forest in Hungary, en Jakucs (ed.) Results of Sikfokut project. Akademi Kiado, Budapest.

TERRADES, J. \& A. ESCARRE (eds.) (1983): Ciclos de nutrientes en ecosistemas terrestres (bosques) estudiados en cuencas de pequeña extensión. Memoria final del proyecto cooperativo 370, 1979-1983, 4 volúmenes, Bellaterra.

VERDÚ, A. M. C. 1984: Circulació de nutrients en tres ecosistemes forestals del Montseny: caiguda de virosta i descomposició de la fullaraca. Tesi Doctoral, Universitat Autonoma de Barcelona, Bellaterra. 